\title{
Perturbations et propriétés holdériennes en traitement de signal
}

\author{
C. Tricot $\left({ }^{1}\right)$, D. Wehbi $\left({ }^{2}\right)$ et C. Roques-Carmes $\left({ }^{2}\right)$ \\ (') Ecole Polytechnique de Montréal, CP 6079, Succursale A. Montréal, Québec, Canada H3C 3A7 \\ ( $\left.{ }^{2}\right)$ Ecole Nationale Supérieure de Mécanique et des Microtechniques (ENSMM), 25030 Besançon Cedex, \\ France
}

(Reçu le 10 novembre 1989, révisé le 28 février 1990, accepté le $1^{\text {er }}$ mars 1990)

\begin{abstract}
Résumé. - La segmentation, en fenêtres temporelles, d'un signal paramétrisé permet de déterminer les perturbations locale et moyenne de ce signal. Des lois du comportement de ces paramètres sont proposées dans le cas d'une part de courbes rectifiables et d'autre part de courbes non rectifiables et fractales. Dans ce dernier cas, la loi trouvée est directement reliée aux propriétés holdériennes locales du signal.

Abstract. - The time parametrization of a signal allows one to define local time windows, in which local perturbations can be measured. The behavior of average local perturbations is shown to be a criterion for the geometric properties of a rectifiable or fractal curve. In the fractal case, we find a hyperbolic law of decrease which is directly related to the local holderian properties of a signal.
\end{abstract}

\section{Introduction.}

Tout traitement de signal, en vue d'applications spécifiques (rugosimétrie bi et tridimensionnelle des surfaces, reconnaissance de l'écriture manuscrite, reconnaissance de la voix, cartographie automatique en géographie, ...), s'appuie nécessairement sur une paramétrisation, implicite ou non, du signal.

L'acquisition des données fournit essentiellement deux types de courbes $\Gamma$ :

- des graphes de fonctions $z(t)$ continues,

- des contours dans le plan, définis en coordonnées cartésiennes par deux fonctions continues $x(t)$ et $y(t)$.

Dans ces notations, $t$ désigne le paramètre. Il peut être interprété comme le temps, la courbe $\Gamma$ étant alors considérée comme une trajectoire.

En d'autres termes, $\Gamma$ est l'image d'un intervalle temporel $[a, b]$ (théoriquement $b$ peut être égal à $+\infty$ ) par une application continue $\gamma$ (la paramétrisation), qui prend ses valeurs dans le plan : à chaque temps $t$, correspond une position $\gamma(t)$, repérée par $(t, z(t))$ dans le cas d'un graphe ou $(x(t), y(t))$ dans le cas d'un contour.

L'image par $\gamma$, d'un sous-intervalle de temps $\left[a_{1}, a_{2}\right]$ est un arc $\gamma\left(a_{1}\right), \gamma\left(a_{2}\right)$ de la courbe $\Gamma$.

Un traitement local consiste en la segmentation du signal en fenêtres temporelles, qui correspond à un partage de $\Gamma$ en une suite de sous-arcs $\Gamma_{1}, \ldots$, $\Gamma_{k}$.
On mesure alors l'irrégularité des sous-arcs dont est composée la courbe et on prend ensuite la moyenne de ces mesures.

Selon le type de mesure, il est possible de tester la rectification locale ou moyenne de $\Gamma$, ou sinon, ses propriétés holdériennes en chaque point (cas d'une courbe non rectifiable et fractale).

\section{La notion de perturbation locale et moyenne.}

Soit un temps $t$ fixé dans l'intervalle temporel $[a, b]$.

Définissons tout d'abord l'arc $A(t, \tau)$ comme l'image par la paramétrisation $\gamma$ de l'intervalle [t $-\tau / 2, t+\tau / 2]$ (cf. Fig. 1). Cet arc est donc un voisinage du point $\gamma(t)$ sur la courbe.

Nous entendons par "perturbé" un arc qui comporte de nombreuses circonvolutions, autrement dit qui paraît remplir le voisinage de $\gamma(t)$.

Nous considérons le segment de droite comme un arc non perturbé. La notion de perturbation est liée à celle d'entropie, ou de chaos. Elle peut se préciser de bien des manières (cf. Fig. 2). Par exemple, la perturbation locale, notée $\varepsilon(t, \tau)$, peut être interprétée dans l'intervalle $[t-\tau / 2, t+\tau / 2]$, par :

$\alpha)$ le diamètre de l'arc $A(t, \tau)$ :

$$
\varepsilon(t, \tau)=\operatorname{diam} A(t, \tau),
$$

B) l'aire $S(t, \tau)$ de l'enveloppe convexe $K(t, \tau)$ de l'arc $A(t, \tau)$, c'est-à-dire la surface formée à 


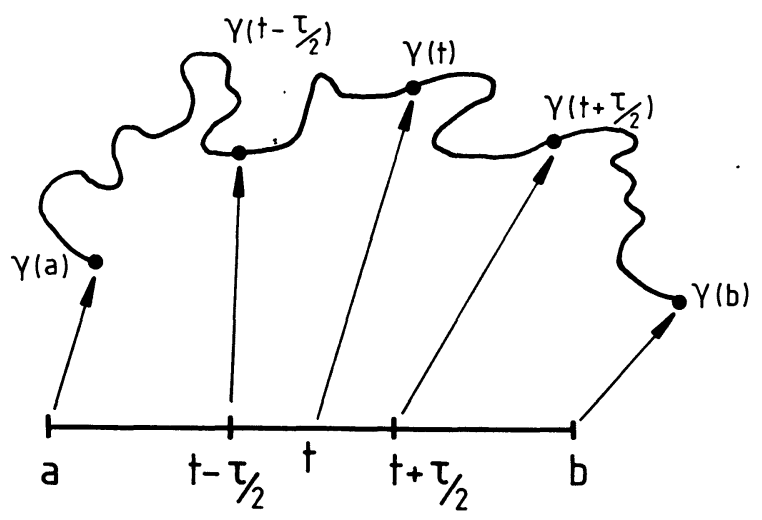

Fig. 1. - Représentation schématisée d'un arc local $A(t, \tau)$ défini par la paramétrisation de la courbe $\Gamma=A(a, b)$.

[Schematic representation of a local arc $A(t, \tau)$ defined on a $\Gamma=A(a, b)$ parametrized curve.]
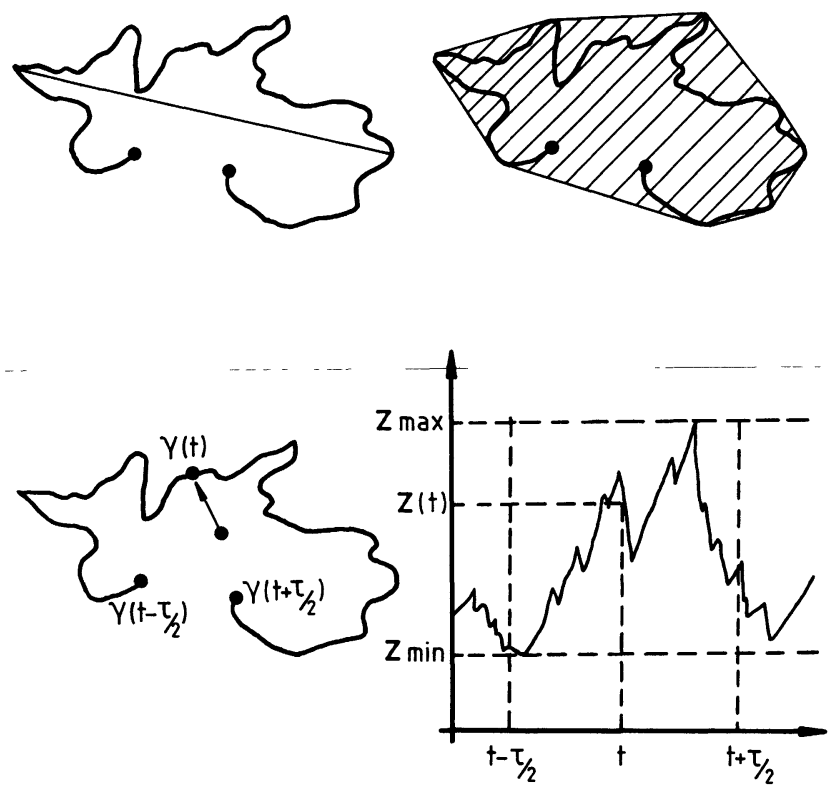

Fig. 2. - Différents procédés d'évaluation de la perturbation locale.

[Different definitions for evaluating the local perturbation of a signal.]

partir de la réunion de tous les segments dont les extrémités sont sur l'arc :

$$
\varepsilon(t, \tau)=S(t, \tau)
$$

$\gamma$ ) la distance entre le point $\gamma(t)$ et le barycentre de l'arc $A(t, \tau)$. Si $\Gamma$ est le graphe d'une fonction $z(t)$, ceci donne :

$$
\varepsilon(t, \tau)=\left|z(t)-\frac{1}{\tau} \int_{t-\tau / 2}^{t+\tau / 2} z(s) \mathrm{d} s\right| .
$$

8) L'oscillation de $\Gamma$ en $t$ :

$$
\varepsilon(t, \tau)=\sup z(x)-\inf z(x)
$$

où

$$
|x-t| \leqslant \tau / 2 \text {. }
$$

$\varepsilon)$ La fonction de structure locale :

$$
\varepsilon(t, \tau)=\frac{1}{\tau} \int_{-\tau / 2}^{+\tau / 2}(z(t)-z(t+s))^{2} \mathrm{~d} s .
$$

A la perturbation locale peut être associée la perturbation moyenne $p(\tau)$, définie par l'intégrale :

$$
p(\tau)=\frac{1}{b-a} \int_{a}^{b} \varepsilon(t, \tau) \mathrm{d} t
$$

si le signal est paramétré sur un intervalle de longueur finie.

Si le signal est défini sur $[0,+\infty]$ alors :

$$
p(\tau)=\lim _{L \rightarrow \infty} \frac{1}{L} \int_{0}^{L} \varepsilon(t, \tau) \mathrm{d} t .
$$

Dans le premier cas, on peut estimer cette intégrale par des sommes de Riemann en réalisant un découpage temporel de la courbe.

On choisit des temps $t_{0}=a, t_{1}, \ldots, t_{N}=b$, équidistants, et l'intégrale est remplacée par la somme :

$$
p(\tau) \approx \frac{1}{N} \sum_{i=0}^{N-1} \varepsilon\left(t_{i}, \tau\right)
$$

ce qui géométriquement, revient à calculer une perturbation moyenne sur une suite de sous-arcs disjoints dont la réunion constitue la courbe $\Gamma$.

\section{Interprétations, applications.}

En reprenant les exemples précédents, la notion de perturbation moyenne peut s'expliquer de la façon suivante :

$\alpha)$ Considérons la courbe polygonale $P_{N}$ dont les segments sont les cordes des arcs $A\left(t_{i}, \tau\right)$. Sa longueur est inférieure à la somme :

$$
\sum_{i=0}^{N-1} \operatorname{diam}\left(A\left(t_{i}, \tau\right)\right)
$$

qui est une évaluation de $N p(\tau) \approx \frac{p(\tau)}{\tau}$.

La perturbation moyenne, divisée par $\tau$, peut donc être interprétée comme une mesure de la longueur de la courbe $\Gamma$, à l'aide d'une jauge de longueur $\tau$.

$\beta)$ La réunion des enveloppes convexes locales $K\left(t_{i}, \tau\right)$ constitue une enveloppe de la courbe $\Gamma$, dont l'aire totale est approximativement $p(\tau) / \tau$. Cette aire est d'autant plus grande que la courbe $\Gamma$ est plus chaotique à l'échelle de la jauge $\tau$. 
$\gamma)$ Ici $p(\tau)$ est l'écart arithmétique moyen, les basses fréquences ayant été éliminées au moyen d'un filtrage par moyenne glissante dont la fenêtre est de largeur $\tau$.

ס) Dans ce cas, la moyenne de l'oscillation est la "variation totale" de la fonction $z(t)$ déjà étudiée dans [1].

$\varepsilon)$ On peut écrire $p(\tau)$ sous la forme :

$$
\begin{aligned}
& p(\tau)=\frac{1}{\tau(b-a)} \times \\
& \times \int_{-\tau / 2}^{+\tau / 2}\left[\int_{a}^{b}(z(t)-z(t+s))^{2} \mathrm{~d} t\right] \mathrm{d} s,
\end{aligned}
$$

après un échange d'intégrales.

C'est donc la moyenne sur $[-\tau / 2, \tau / 2]$ de la fonction de structure $S(s)$ évaluée sur le domaine de définition de $z$ :

$$
p(\tau)=\frac{1}{\tau(b-a)} \int_{-\tau / 2}^{+\tau / 2} S(s) \mathrm{d} s .
$$

On remarquera que, lorsque le signal est continu, la perturbation moyenne tend vers 0 lorsque $\tau$ tend vers 0 .

La loi de décroissance de $p(\tau)$ est une caractéristique importante du signal observé. Nous allons étudier deux cas importants, en reprenant les données précédentes.

\subsection{CAS DES COURBES RECTIFIABLES ( $\Gamma$ EST DE LONGUEUR FINIE).}

$\alpha)$ Dans ce cas $p(\tau) / \tau$ tend vers une limite finie $C$. Cette limite est en fait égale à la vitesse moyenne lorsque $\Gamma$ est considérée comme une trajectoire.

Autrement dit $p(\tau)$ est équivalent à $C \tau$ au voisinage de 0 .

$\beta)$ Dans ce cas, $p(\tau) / \tau^{2}$ tend vers 0 . En effet, chaque $\varepsilon(t, \tau)$ est l'aire d'un ensemble convexe qui devient de plus en plus « aplati » lorsque $\tau$ tend vers 0 . Cette aire tend vers 0 plus vite que le carré de son diamètre. Dans le cas limite où $\tau$ est une ligne droite, $p(\tau)=0$ pour tout $\tau$.

$\gamma)$ Lorsque $\Gamma$ est le graphe d'une fonction continue $z(t)$, cette fonction est dérivable plusieurs fois presque partout. En prenant le développement de Taylor de $z(s)$ au voisinage de $t$, on trouve après une intégration :

$$
\varepsilon(t, \tau) \approx C \tau^{2}
$$

où $C$ dépend de la dérivée seconde de $z$ en $t$.

Autrement dit, le rapport $p(\tau) / \tau^{2}$ tendra vers une limite finie.

$\delta$ ) Pour tout $t$, le rapport $\varepsilon(t, \tau) / \tau$ tend vers la dérivée de la fonction $z$. La loi de décroissance de la perturbation moyenne est :

$$
p(\tau) \approx C \tau .
$$

ع) Un calcul analogue au cas $(\gamma)$ donne :

$$
\varepsilon(t, \tau) \approx\left(\tau z^{\prime}(t)\right)^{2}
$$

et donc $p(\tau) \approx C \tau^{2}$.

On a donc trouvé des décroissances en $\tau$ ou en $\tau^{2}$ pour caractériser la perturbation moyenne. Notons que l'on peut qualifier les courbes rectifiables par des lois en $\tau^{n}$ où $n$ est entier.

3.2 CAS Des Courbes NON ReCtifiables. - Parmi toute la famille des courbes non rectifiables, les courbes fractales se recommandent par la propriété intrinsèque suivante : lorsque $\tau$ tend vers zéro, la perturbation locale $\varepsilon(t, \tau)$ tend vers zéro suivant une loi de puissance en $\alpha$ indépendante de $t$.

Le paramètre $\alpha$ est alors fonction de la dimension fractale $\Delta(\Gamma)$ de la courbe.

On peut distinguer deux cas importants de courbes fractales :

a) Les courbes possédant une structure de similitude interne, où chaque arc est une image de la courbe totale par une similitude. La paramétrisation est généralement induite par la construction même de la courbe.

Deux applications sont proposées en se référant aux cas présentés précédemment.

$\alpha)$ Un arc de mesure $\tau$ a un diamètre de l'ordre de $\tau^{\alpha}$ où $\alpha=1 / \Delta(\Gamma)$ [2]. Dans ce cas, la perturbation moyenne suit une loi :

$$
p(\tau) \approx \tau^{1 / \Delta(\Gamma)} .
$$

Lorsque $\Delta(\Gamma) \rightarrow 1$ ( $\Gamma$ tend vers une courbe rectifiable) cette loi devient $p(\tau) \approx \tau$ ce qui correspond à notre calcul antérieur dans le cas rectifiable.

$\beta)$ L'enveloppe de $\Gamma$ constituée de la réunion des enveloppes convexes locales a une aire de l'ordre de :

$$
\tau^{(2 / \Delta(\Gamma))-1}
$$

(comme l'aire de la «saucisse de Minkowski ») [3].

C'est aussi l'ordre de grandeur de $p^{*}(\tau) / \tau$. Donc $p(\tau)$ suit une loi de puissance en $\alpha$, avec $\alpha=$ $2 / \Delta(\Gamma)$.

b) Les graphes de fonctions $z(t)$, définis sur $[0, \infty]$ possédant une structure d'affinité interne.

Ils sont caractérisés par une propriété holdérienne d'exposant $H, 0<H<1$, qui se traduit par l'égalité pour tout $t$ :

$$
z(b t)=b^{H} z(t),
$$

où $b$ est un paramètre caractéristique de $z$ dans le cas de l'affinité interne stricte. Dans le cas de l'affinité interne statistique, cette égalité signifie seulement l'identité des distributions de probabilité, et $b$ possède une valeur arbitraire. 


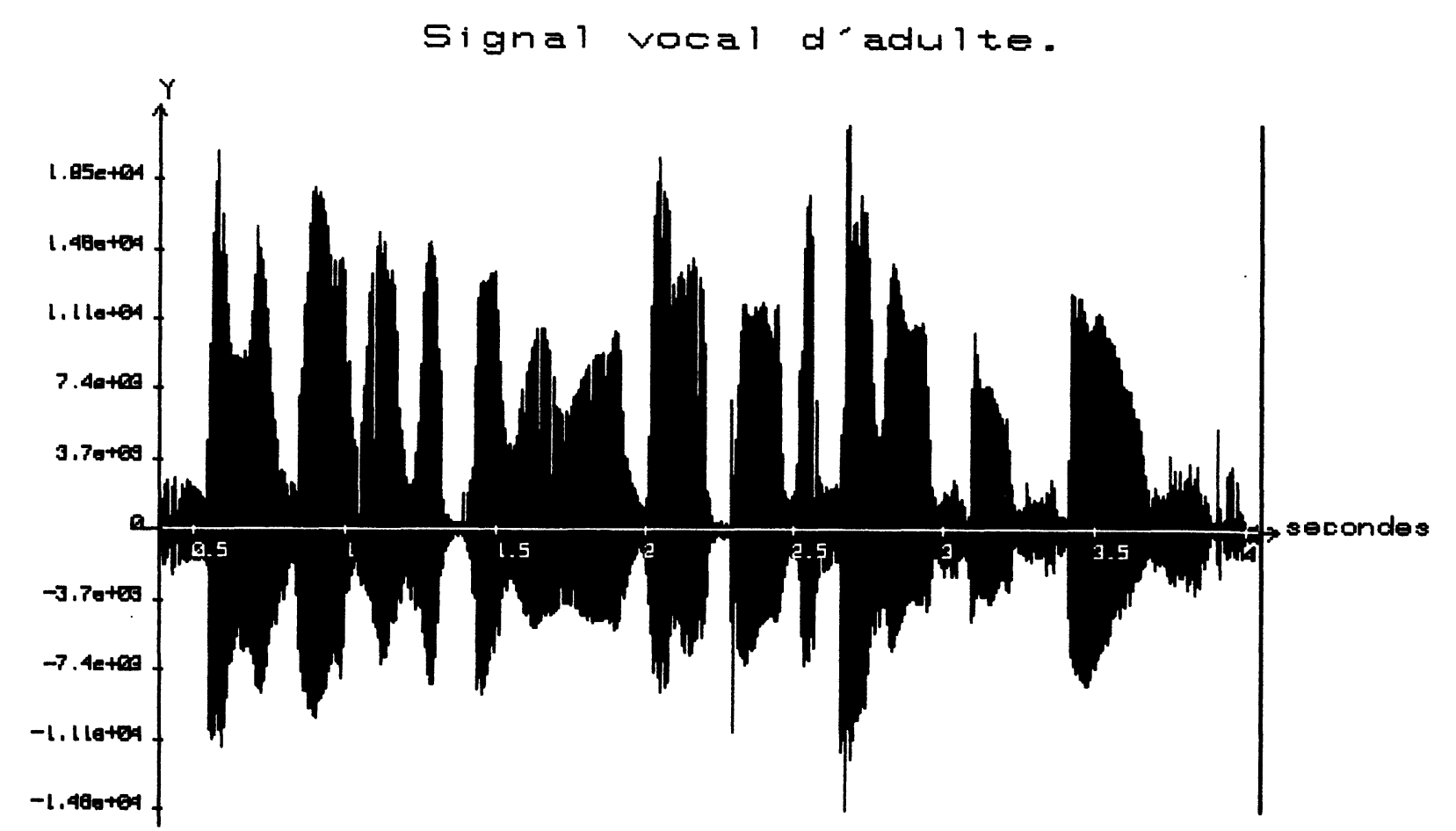

Fig. 3. - Exemple de signal vocal extrait d'une phrase prononcée par un adulte. L'oscillogramme présenté illustre la variation de l'amplitude du son émis pendant une durée de 3,674 s.

[Example of a vocal signal.]

$$
\text { Signal d'un cri de bebe. }
$$

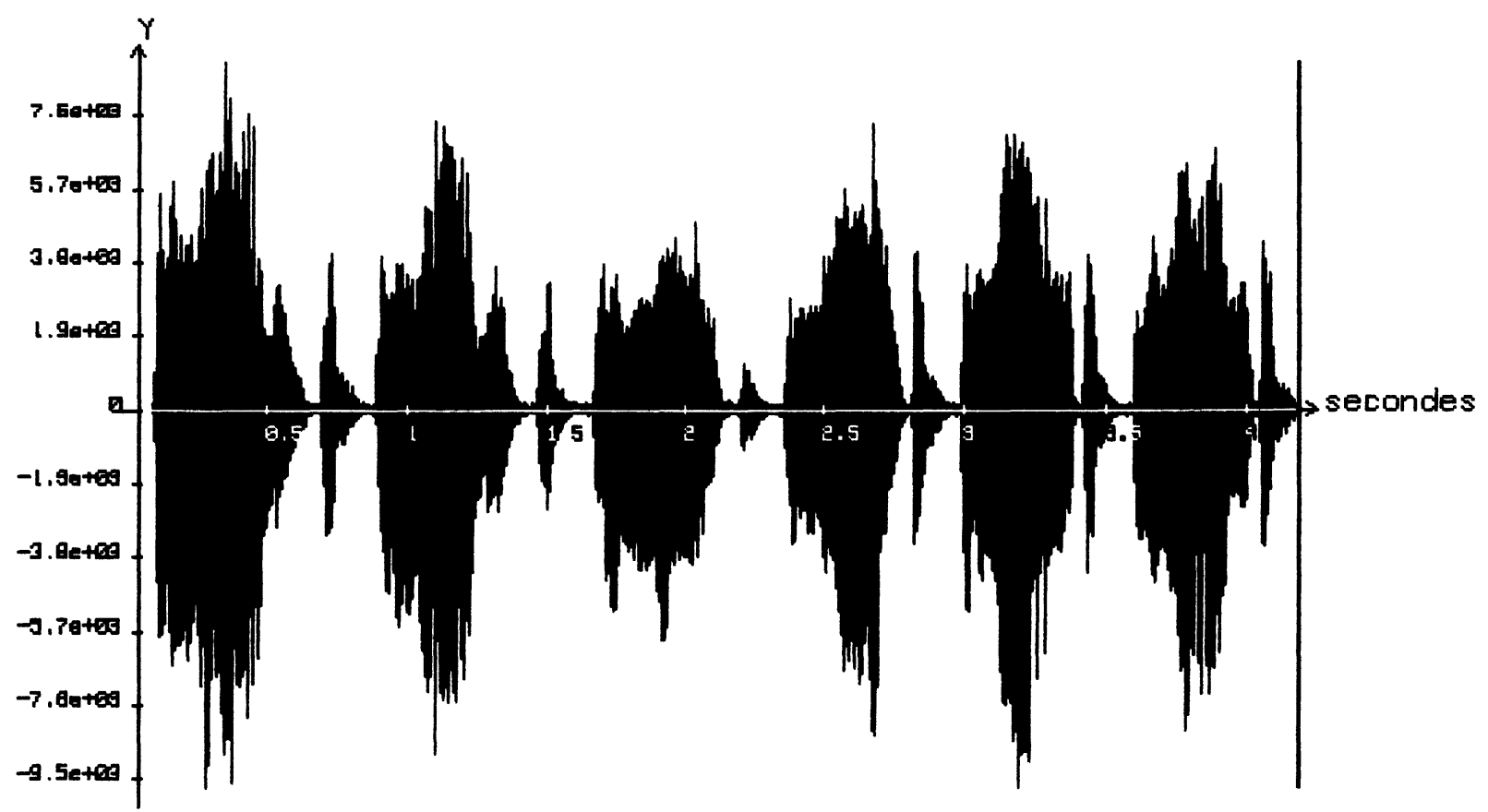

Fig. 4. - Signal acoustique d'une séquence de cris d'un nouveau-né (4 jours). L'oscillogramme présenté illustre la variation de l'amplitude du son émis pendant une durée de 4,174s.

[Signal representing an infant cry.] 


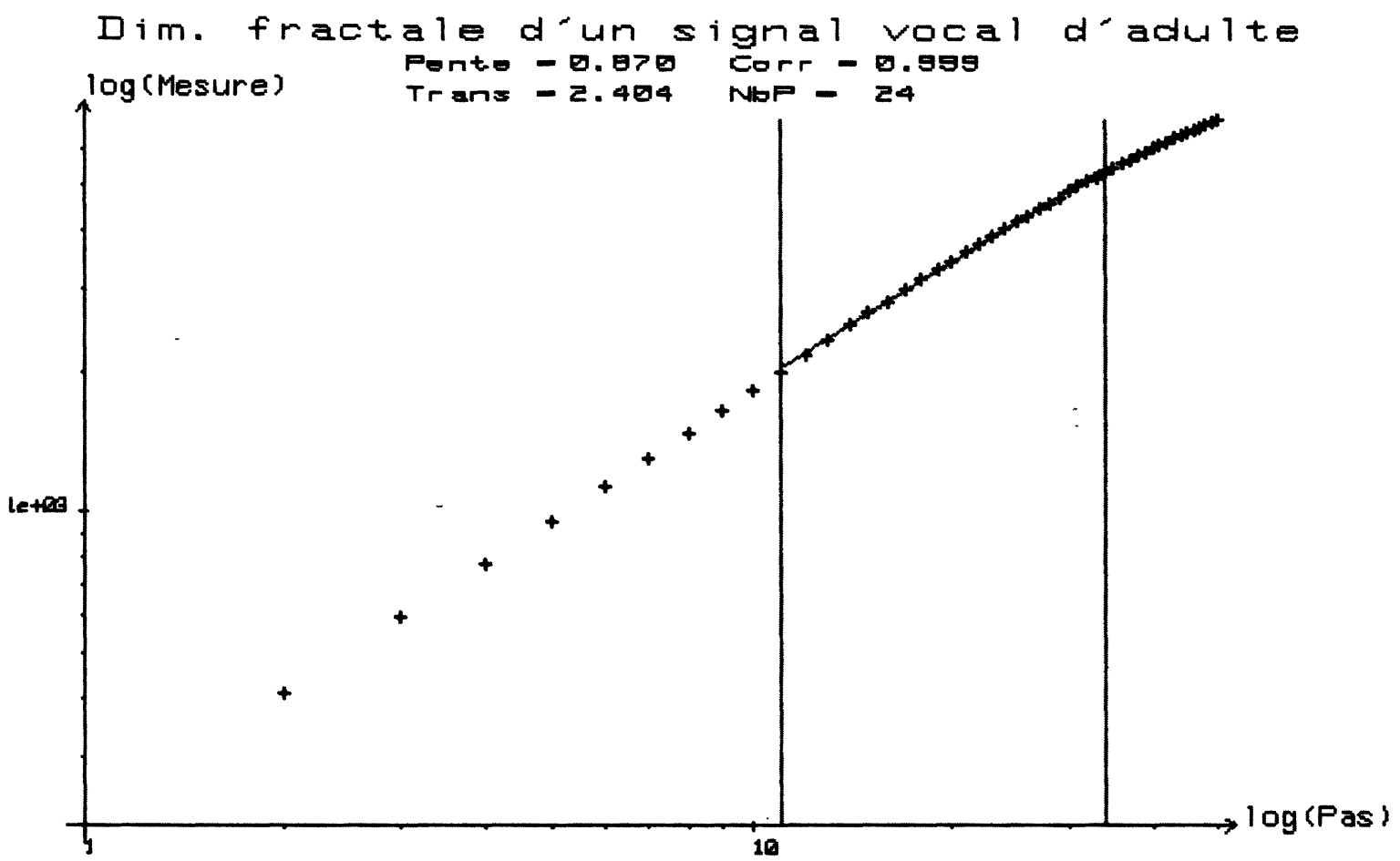

Fig. 5. - Détermination de la dimension fractale du signal vocal de la figure 3 à l'aide de la méthode de variation (paragraphe 3.8). L'abscisse "pas », variant de 1 à 50, représente la demi-largeur de l'intervalle $\tau$ exprimée en nombre de pas d'échantillonnage. Le pas d'échantillonnage étant dans ce cas égal à $0,05 \mathrm{~ms}, \tau$ varie de $0,1 \mathrm{~ms}$ à $5 \mathrm{~ms}$.

[Determination of the fractal dimension of the vocal signal presented in figure 3.]

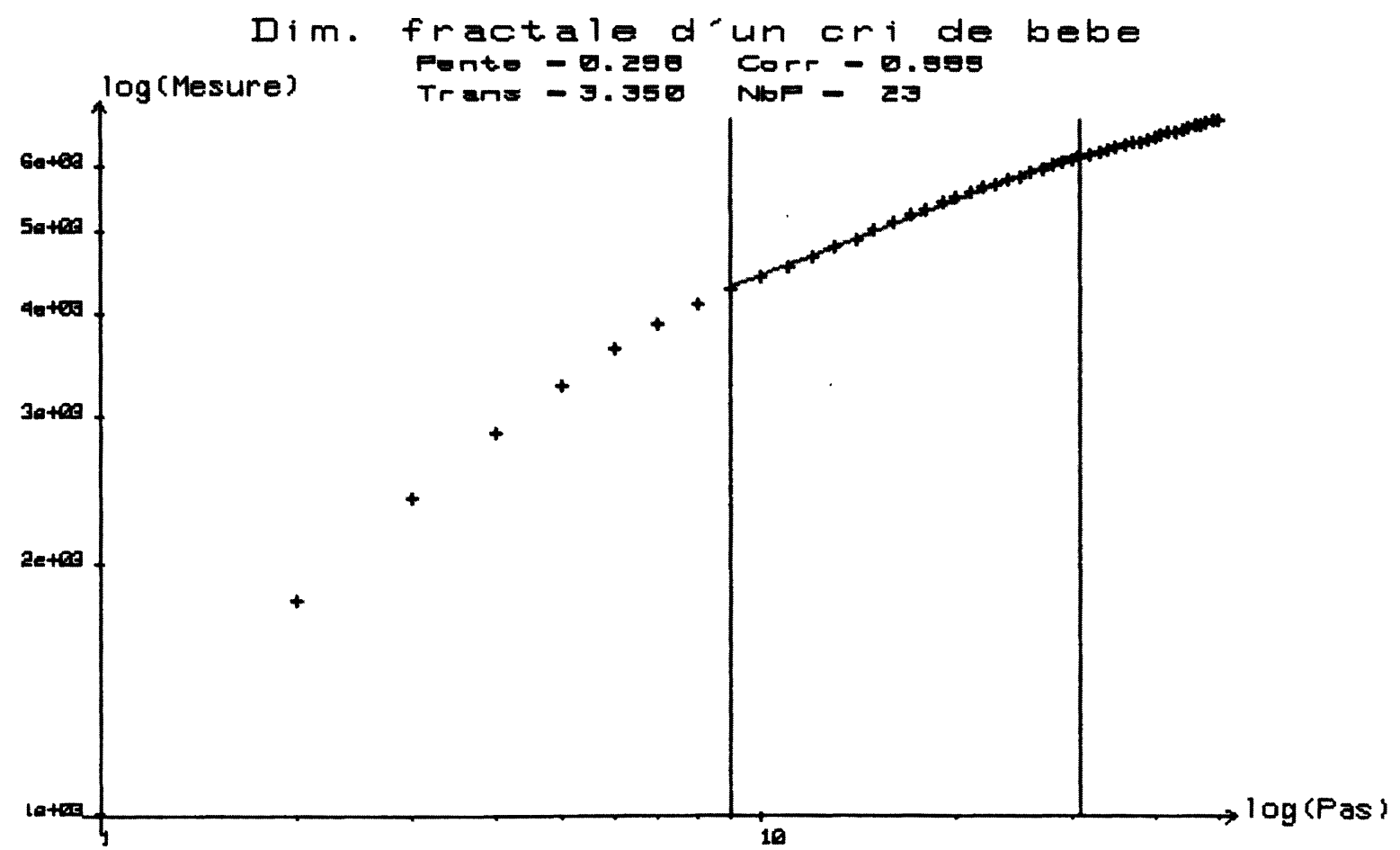

Fig. 6. - Détermination de la dimension fractale du signal vocal de la figure 4 à l'aide de la méthode de variation

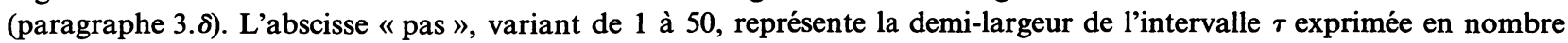
de pas d'échantillonnage. Le pas d'échantillonnage étant dans ce cas égal à $0,05 \mathrm{~ms}, \tau$ varie de $0,1 \mathrm{~ms}$ à $5 \mathrm{~ms}$.

[Determination of the fractal dimension of the infant cry signal presented in figure 4.] 


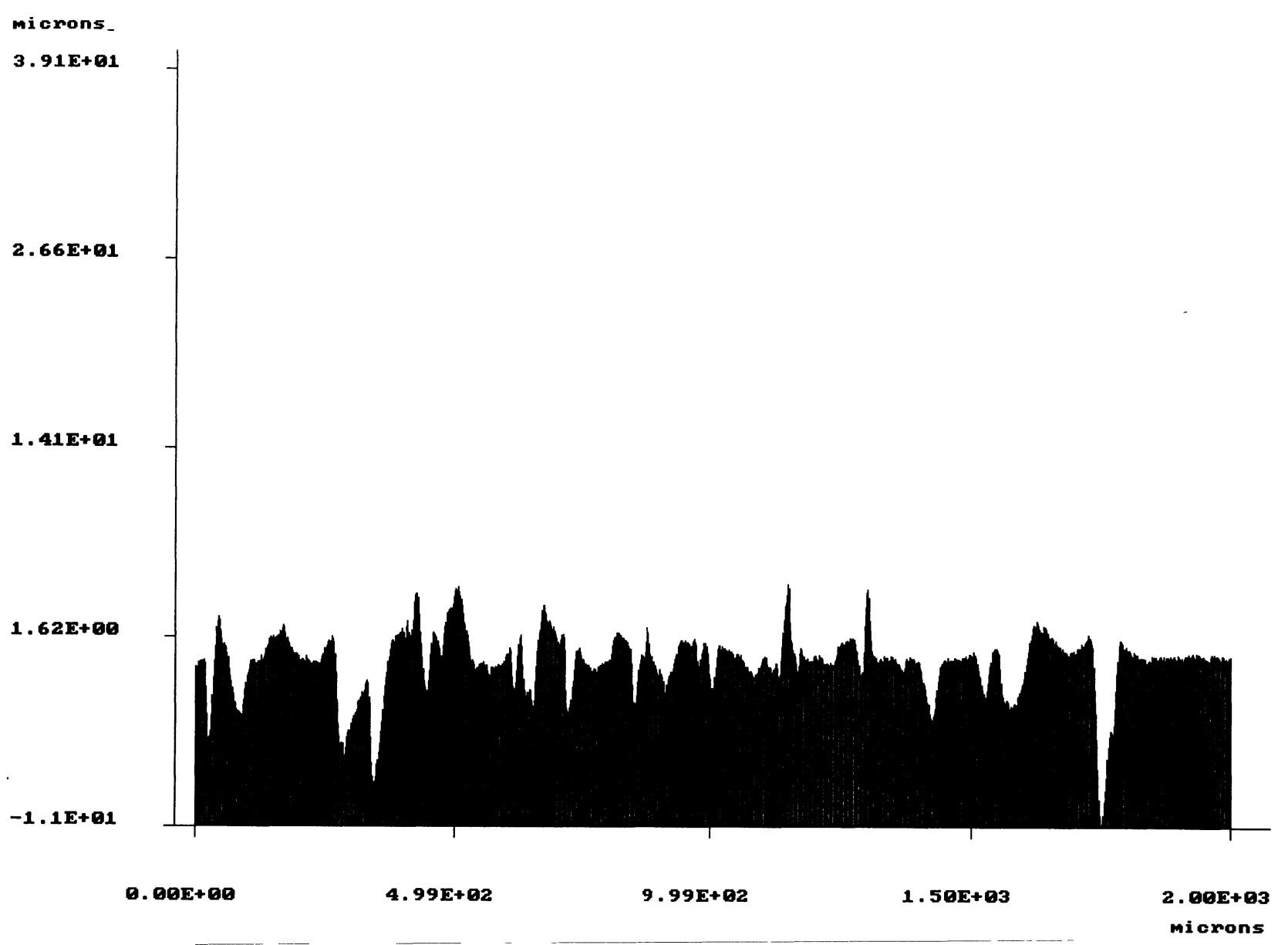

Fig. 7. - Exemple de profil de rugosité d'une surface sablée de taiton. Le profil présenté illustre la variation de l'amplitude de rugosité sur une longueur d'évaluation de $2 \mathrm{~mm}$. Echelle d'amplitude : 1 division représente 12,5 $\mu \mathrm{m}$.

[Surface roughness profile of a sand blasted brass sample.]

On a déjà montré [4] que l'exposant $H$ était dans ce cas égal à :

$$
2-\Delta(\Gamma)
$$

Pour trouver la loi de décroissance vers 0 de la perturbation $p(\tau)$ il suffit de trouver un paramètre $\alpha$ tel que :

$$
p(b \tau)=b^{\alpha} p(\tau) .
$$

La fonction $p(\tau)$ est alors équivalente à $\tau^{\alpha}$ au voisinage de 0 [5].

Des données de base sont proposées en prenant comme exemple les cas traités précédemment.

$\gamma)$ On montre d'abord que

$$
\varepsilon(b t, b \tau)=b^{H} \varepsilon(t, \tau),
$$

par simple changement de variable.

D'où l'on tire :

$$
p(b \tau)=\lim _{L \rightarrow \infty} \frac{1}{L} \int_{0}^{L} \varepsilon(t, b \tau) \mathrm{d} t=b^{H} p(\tau) .
$$

La loi de $p(\tau)$ est donc une loi en :

$$
\tau^{H}=\tau^{2-\Delta(\Gamma)} .
$$

Remarquons que, cette fois-ci, en faisant tendre $\Delta(\Gamma)$ vers 1 , on ne retrouve pas pour la perturbation le comportement rectifiable $p(\tau) \approx \tau^{2}$ qui est comparable au rayon de courbure. Pour expliquer ce fait on retiendra que dans le cas non rectifiable, la structure chaotique de la courbe augmente la distance entre $\Gamma$ et la courbe rectifiable obtenue par filtrage passe-bas de $\Gamma$.

$\delta$ ) Le calcul est le même que précédemment :

$$
\varepsilon(b t, b \tau)=b^{H} \varepsilon(t, \tau) .
$$

Il en résulte un comportement de $p(\tau)$ en $\tau^{2-\Delta(\Gamma)}$ [6].

ع) Ici :

$$
\varepsilon(b t, b \tau)=b^{2 H} \varepsilon(t, \tau)
$$

Ce qui donne :

$$
p(b, \tau)=b^{2 H} p(\tau),
$$




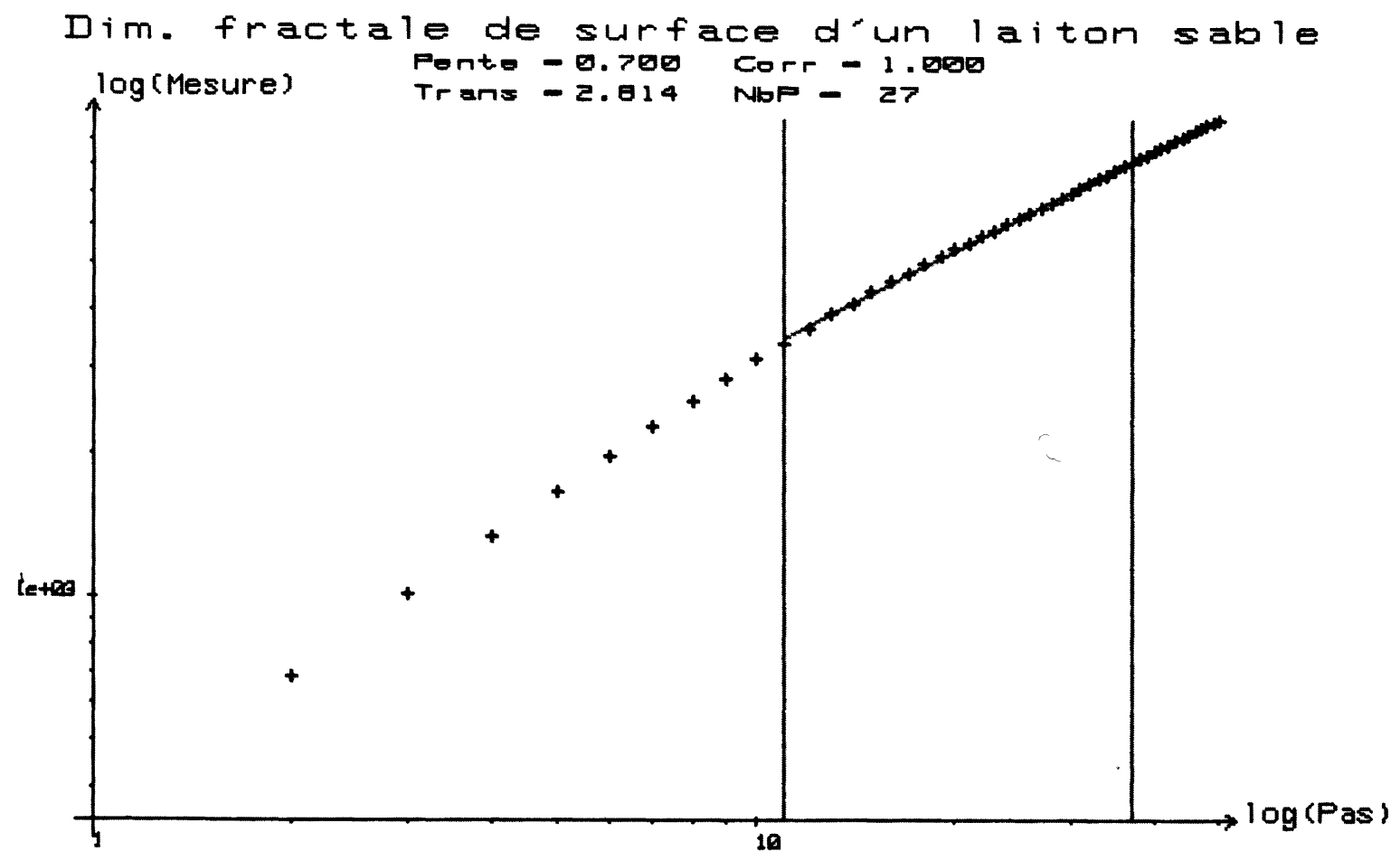

Fig. 8. - Détermination de la dimension fractale du signal rugosimétrique présenté sur la figure 7 à l'aide de la méthode de variation (paragraphe 3.8). L'abscisse "pas ", variant de 1 à 50 , représente la demi-largeur de l'intervalle $\tau$ exprimée en nombre de pas d'échantillonnage. Le pas d'échantillonnage étant dans ce cas égal à $1 \mu \mathrm{m}, \tau$ varie de $2 \mu \mathrm{m}$ à $100 \mu \mathrm{m}$.

[Determination of the fractal dimension of the roughness profile presented in figure 7.]

et donc

$$
p(\tau)=\tau^{2 H}=\tau^{4-2 \Delta(\Gamma)}
$$

au voisinage de 0 .

A titre d'illustration, les figures 3 à 8 montrent différents types de résultats sur des signaux $z(t)$ (signal vocal) et $z(x)$ (profil de rugosité).

En utilisant la méthode $\delta$, l'exemple d'un signal vocal de parole (cf. Fig. 3) éclaire de façon privilégiée la relation complexe qui existe entre les formes acoustiques de base des sons parlés et leur transformation au sein d'une chaîne sonore.

La distribution de l'énergie sonore au cours du temps de parole varie en fonction d'un très grand nombre de paramètres : la voix de l'individu, le type de phrase, le type de sons, le type de discours...

Ce qui caractérise la parole par rapport au cri d'un nouveau-né, par exemple, c'est la variabilité permanente du signal acoustique. La figure 4 (cri du bébé) fait clairement apparaître la périodicité des phases d'inspiration et d'expiration "criées ". On ne rencontre jamais cette forme globale périodique dans le cas de signaux de parole.

Une approche holdérienne des deux types de signaux permet en revanche de caractériser un signal de parole et un cri de bébé (Figs. 5 et 6). Elle prend en compte à la fois les perturbations locales et les perturbations globales de chacun des signaux. Une étude détaillée concernant l'identification de l'individu par la connaissance d'invariants est en cours de réalisation [7].

L'exemple d'un profil de rugosité (cf. Fig. 7) permet de qualifier les dénivelées de surface en termes de perturbations ; c'est à ce titre une méthode originale qui trouve de nombreuses applications en Science des Matériaux.

Elle complète harmonieusement la description classique des profils $z(x)$ en termes de critères statistiques d'amplitude et de paramètres spectraux.

La détermination de la dimension fractale d'un tel signal est illustrée sur la figure 8 .

\section{Conclusions.}

On retiendra tout d'abord les points importants suivants :

- un signal bien défini est un signal paramétré ;

- paramétriser une courbe est équivalent à considérer celle-ci comme le support d'une mesure ;

- la mesure permet de déterminer des fenêtres temporelles sur la courbe;

- dans chacune des fenêtres, on peut calculer une perturbation locale $\varepsilon(t, \tau)$;

- on en déduit une perturbation moyenne $p(\tau)$ qui caractérise l'irrégularité globale de la courbe. 
En partant de ce principe, nous avons proposé une liste de définitions possibles pour la perturbation dont certaines s'appliquent aux contours (courbes de niveaux, frontières d'agrégats, ...) et d'autres aux graphes de fonctions continues $z(t)$.

Parmi les premières, la méthode des diamètres d'arcs, semble la plus simple et la plus performante à ce jour.

Parmi les secondes, les méthodes utilisant les paramètres "rugosité moyenne" et "fonction de structure " donnent les résultats les plus probants.
On retiendra de cette nouvelle analyse des signaux, son aspect plus essentiel, la loi de convergence de $p(\tau)$ vers 0 , qui caractérise la structure interne du signal.

Dans le cas non rectifiable et fractal, cette loi est en $\tau^{\alpha}$, où $\alpha$ est relié directement aux propriétés holdériennes locales de $z$, donc à la dimension fractale de son graphe.

Des applications pratiques quantifiant l'oscillation et la variation totale des signaux type $z(t)$ ou $z(x)$ ont été présentées.

\section{Bibliographie}

[1] Tricot C., Roques-Carmes C., Quiniou J.-F., WehBi D., Dubuc B., Evaluation de la dimension fractale d'un graphe, Revue Phys. Appl. 23 (1988) 111-124.

[2] Mandelbrot B., The Fractal Geometry of Nature (Freeman) 1982.

[3] Tricot C., Wehbi D., Quiniou J.-F., RoQues-CarMES C., Concepts of non-integer dimension applied to image treatment. Acta Stereologica 6/III (1987) 839-844.
[4] MANDelbrot B., Self-affine fractals and fractal dimension, Phys. Scr. 32 (1986) 257-260.

[5] Tricot C., Dimension fractale et spectre, J. Chim. Phys. 85 (1988) 379-385.

[6] Tricot C., Dubuc B., Variation d'une fonction et dimension de son graphe, C. R. Acad. Sc. Paris 306 (1988) 531-533.

[7] Wehbi D., Roques-Carmes C., Lhote E., En cours de rédaction pour publication dans la Rev. Phon. 